\title{
A FAMILY OF TYPE III EXTENSIONS OF THE TRACE ON THE CHOI ALGEBRA
}

\author{
P. J. STACEY
}

(Communicated by Palle E. T. Jorgensen)

\begin{abstract}
An infinite family of nonequivalent type III factor states of $\mathrm{O}_{2}$ is constructed, each one of which extends the trace on the Choi algebra.
\end{abstract}

\section{INTRODUCTION}

In [7] the authors show how the trace on the Choi algebra can be extended to a pure state of the Cuntz algebra $\mathrm{O}_{2}$; they also remark that the trace on the Choi algebra can be extended to a state of type $\mathrm{III}_{1 / 2}$ and they pose the problem of producing extensions of type $\mathrm{II}_{\infty}$ and type $\mathrm{III}_{\lambda}\left(\lambda \neq \frac{1}{2}\right)$. In the present note a countable infinity of nonisomorphic $\mathrm{III}_{\lambda}$ extensions is produced.

This paper was written in late 1988 but was not published at that stage because the author was informed of the existence of the prior (but then unpublished) independent related papers [2,9]. It has since emerged that there is sufficient interest in the alternative approach adopted in this paper to merit publication. The exact relationship of the present results to those of $[2,9]$ will be made precise in the sequel.

The Cuntz algebra $\mathrm{O}_{2}$ is an infinite simple $C^{*}$-algebra generated by two isometries $S_{1}, S_{2}$, satisfying $S_{1}^{*} S_{1}=S_{2}^{*} S_{2}=1$ and $S_{1} S_{1}^{*}+S_{2} S_{2}^{*}=1$. It is shown in [5] that there is a faithful conditional expectation $E$ from $0_{2}$ onto the $C^{*}$-subalgebra $U_{2}$ generated by elements of the form $S_{\mu_{1}} S_{\mu_{2}} \cdots S_{\mu_{r}} S_{\nu_{r}}^{*} \cdots S_{\nu_{2}}^{*} S_{\nu_{1}}^{*}$ and that there is a $*$-isomorphism $\theta$ from $U_{2}$ onto the $U H F$ algebra $U H F\left(2^{\infty}\right)=\bigotimes_{i=1}^{\infty} M_{2}(\mathbf{C})$ such that $\theta\left(S_{\mu_{1}} \cdots S_{\mu_{r}} S_{\nu_{r}}^{*} \cdots S_{\nu_{1}}^{*}\right)=e_{\mu_{1} \nu_{1}} \otimes \cdots \otimes e_{\mu_{r} \nu_{r}} \otimes$ 1 , where $e_{i j}$ are the obvious matrix units in $M_{2}(\mathbf{C})$.

The Choi algebra, introduced in [3], can be regarded as the $C^{*}$-subalgebra of $0_{2}$ generated by the finite order unitaries $u=S_{1} S_{2}^{*}+S_{2} S_{1}^{*}$ and $v=S_{1} S_{2}^{* 2}+$ $S_{2} S_{1} S_{1}^{*}+S_{2}^{2} S_{1}^{*} S_{2}^{*}$ (for which $u^{2}=v^{3}=1$ ). It possesses a unique trace $\tau$, which is specified on the finite linear combinations of reduced words in $u$ and $v$ by $\tau\left(\alpha 1+\sum \alpha_{i} \omega_{i}\right)=\alpha$ for $\alpha, \alpha_{i} \in \mathbf{C}$ and $\omega_{i}$ a nontrivial reduced word in $u, v$. (See [3, Proposition 2.3] for details.)

\section{Extensions of the trace on the Choi Algebra}

For each $i$ let $\varphi_{i}$ be a state on $M_{2}(\mathbf{C})$ such that $\varphi_{i}\left(e_{12}\right)=\varphi_{i}\left(e_{21}\right)=0$.

Received by the editors June 13, 1990.

1980 Mathematics Subject Classification (1985 Revision). Primary 46L30, 46L35. 
The purpose of this section is to show that $\left(\bigotimes_{i=1}^{\infty} \varphi_{i}\right) \circ \theta \circ E$ restricts to the trace on the Choi algebra, where $E: 0_{2} \rightarrow U_{2}$ and $\theta: U_{2} \rightarrow \bigotimes_{i=1}^{\infty} M_{2}(\mathbf{C})$ were introduced in $\S 1$.

Lemma 2.1. Let $u=S_{1} S_{2}^{*}+S_{2} S_{1}^{*}$ and let $v=S_{1} S_{2}^{* 2}+S_{2} S_{1} S_{1}^{*}+S_{2}^{2} S_{1}^{*} S_{2}^{*}$.

(i) $u v=S_{1}^{2} S_{1}^{*}+S_{1} S_{2} S_{1}^{*} S_{2}^{*}+S_{2} S_{2}^{* 2}$.

(ii) $u v^{2}=S_{1} S_{2} S_{1}^{*}+S_{1}^{2} S_{2}^{* 2}+S_{2} S_{1}^{*} S_{2}^{*}$.

(iii) Any nontrivial word in $u v$ and $u v^{2}$ is of the form $S_{1} \omega_{11} S_{1}^{*}+S_{1} \omega_{12} S_{2}^{*}+$ $S_{2} \omega_{22} S_{2}^{*}$, where $\omega_{11}$ is a nontrivial word in $S_{1}, S_{2}, \omega_{22}$ is a nontrivial word in $S_{1}^{*}, S_{2}^{*}$, and $\omega_{12}$ is a sum of words of the form $a S_{1} S_{2}^{*} b$ and $c S_{2} S_{1}^{*} d$, where $a, c$ are (possibly empty) words in $S_{1}, S_{2}$ and $b, d$ are (possibly empty) words in $S_{1}^{*}, S_{2}^{*}$.

Proof. (i) and (ii). These result from elementary computations.

(iii) This is proved inductively by noting that

$$
\begin{aligned}
& \left(S_{1} \omega_{11} S_{1}^{*}+S_{1} \omega_{12} S_{2}^{*}+S_{2} \omega_{22} S_{2}^{*}\right) u v \\
& \quad=S_{1} \omega_{11} S_{1} S_{1}^{*}+S_{1}\left(\omega_{11} S_{2} S_{1}^{*}+\omega_{12} S_{2}^{*}\right) S_{2}^{*}+S_{2} \omega_{22} S_{2}^{* 2}
\end{aligned}
$$

and

$$
\begin{aligned}
& \left(S_{1} \omega_{11} S_{1}^{*}+S_{1} \omega_{12} S_{2}^{*}+S_{2} \omega_{22} S_{2}^{*}\right) u v^{2} \\
& \quad=S_{1} \omega_{11} S_{2} S_{1}^{*}+S_{1}\left(\omega_{11} S_{1} S_{2}^{*}+\omega_{12} S_{1}^{*}\right) S_{2}^{*}+S_{2} \omega_{22} S_{1}^{*} S_{2}^{*},
\end{aligned}
$$

which are both of the required form.

Proposition 2.2. For each $i$ let $\varphi_{i}$ be a state on $M_{2}(\mathbf{C})$ such that $\varphi_{i}\left(e_{12}\right)=$ $\varphi_{i}\left(e_{21}\right)=0$, let $E: 0_{2} \rightarrow U_{2}$ be the canonical conditional expectation and let $\theta: U_{2} \rightarrow \bigotimes_{i=1}^{\infty} M_{2}(\mathbf{C})$ be the isomorphism specified by $\theta\left(S_{\mu_{1}} \cdots S_{\mu_{r}} S_{\nu_{r}}^{*} \cdots S_{\nu_{1}}^{*}\right)=$ $e_{\mu_{1} \nu_{1}} \otimes \cdots \otimes e_{\mu_{r} \nu_{r}} \otimes 1$. Then $\psi=\left(\otimes_{i=1}^{\infty} \varphi_{i}\right) \circ \theta \circ E$ restricts to the trace on the Choi algebra.

Proof. From [3, Proposition 2.3] (and its proof) it suffices to show that $\psi(\omega)=$ 0 for each nontrivial reduced word $\omega$ in $u, v$. However any nontrivial reduced word $\omega$ in $u, v$ is of the form $x, v x, v^{2} x, x u, v x u$, or $v^{2} x u$ where $x$ is a word in $u v$ and $u v^{2}$. Then, using Lemma 2.1,

$$
\begin{aligned}
E(x) & =S_{1} \omega_{12} S_{2}^{*}, & E(v x) & =S_{2} \omega_{12} S_{2}^{*}, \\
E\left(v^{2} x\right) & =S_{2} \omega_{12} S_{2}^{*}, & E(x u) & =S_{1} \omega_{12} S_{1}^{*}, \\
E(v x u) & =S_{2} \omega_{12} S_{1}^{*}, & E\left(v^{2} x u\right) & =S_{2} \omega_{12} S_{1}^{*},
\end{aligned}
$$

where $\omega_{12}$ is a (possibly empty) sum of words of the form $S_{\mu_{1}} \cdots S_{\mu_{r}} S_{1} S_{2}^{*} S_{\nu_{r}}^{*} \cdots$ $S_{\nu_{1}}^{*}$ or $S_{\mu_{1}} \cdots S_{\mu_{r}} S_{2} S_{1}^{*} S_{\nu_{r}}^{*} \cdots S_{\nu_{1}}^{*}$. Each summand of $E(\omega)$ therefore corresponds under $\theta$ to a matrix unit $e_{i j} \otimes e_{\mu_{1} \nu_{1}} \otimes \cdots e_{\mu_{r} \nu_{r}} \otimes e_{k l} \otimes 1$, where $e_{k l}=e_{21}$ or $e_{k l}=e_{12}$, from which it follows that $\left(\bigotimes_{i=1}^{\infty} \varphi_{i}\right) \circ \theta \circ E(\omega)=0$, as required.

Proposition 2.2 can also be obtained as a consequence of [2, Proposition 2.2] or [9, Theorem 2.8]; both of these results are proved by elementary combinatorial arguments, similar to those used here.

\section{A FAMILY OF TYPE III STATES ON $\mathrm{O}_{2}$}

For each $0<\mu<\frac{1}{2}$ let $\rho_{\mu}$ be the state on $M_{2}(\mathbf{C})$ defined by $\rho_{\mu}\left(\sum e_{i j} a_{i j}\right)=$ $\mu a_{11}+(1-\mu) a_{22}$, let $\varphi_{\mu}$ be the state $\left(\otimes_{i=1}^{\infty} \rho_{\mu}\right) \circ \theta$ on $U_{2}$ and let $\psi_{\mu}$ be the state 
$\varphi_{\mu} \circ E$ on $0_{2}$, where $E$ and $\theta$ are as in $\S 2$. Note that, in the language of [6], $\psi_{\mu}$ is the quasi-free state of $0_{2}$ corresponding to the matrix $K=\mu e_{11}+(1-\mu) e_{22}$ and that, as remarked before [6, Proposition 2.2], $\psi_{\mu}$ is a factor state. In order to determine its isomorphism type we will consider the invariants $S(M)$ and $T(M)$ (where $M=\pi_{\psi_{\mu}}\left(0_{2}\right)^{\prime \prime}$ ) introduced by Connes in [4].

Lemma 3.1. Let $M=\pi_{\psi_{\mu}}\left(0_{2}\right)^{\prime \prime}$. Then, for each $0<\mu<\frac{1}{2},\{\mu, 1-\mu\} \subseteq S(M)$. Proof. By [6, Proposition 2.2] $\psi_{\mu}$ is a $K M S$ state at inverse temperature 1 for the automorphism group $\left\{\alpha_{t}: t \in \mathbf{R}\right\}$ of $0_{2}$ specified by $\alpha_{t}\left(S_{1}\right)=\mu^{-i t} S_{1}$ and $\alpha_{t}\left(S_{2}\right)=(1-\mu)^{-i t} S_{2}$ for each $t \in \mathbf{R}$. By [8, 8.12.4] $\psi_{\mu}$ is $\alpha$-invariant (as can also be seen directly) and hence, by [8,7.4.12 and 7.4.10], $\alpha$ extends to a covariant action of $\mathbf{R}$ on $M$ which, by $[8,8.14 .3]$, is the modular automorphism group $\left\{\sigma_{t}^{\mu}: t \in \mathbf{R}\right\}$ of $M$ associated with the faithful normal extension of $\psi_{\mu}$ to $M$.

For each nonzero projection $e$ in the fixed point algebra for $\sigma^{\mu}$ let $\left(\sigma^{\mu}\right)^{e}$ denote the restriction of $\sigma^{\mu}$ to $e M e$, and let

$$
S_{p}\left(\left(\sigma^{\mu}\right)^{e}\right)=\bigcap_{f \in F_{\mu}}\left\{\lambda \in \mathbf{R}^{+}: \int \lambda^{-i t} f(t) d t=0\right\},
$$

where $F_{\mu}=\left\{f \in L_{1}(\mathbf{R}): \int f(t) \sigma_{t}^{\mu}(x) d t=0\right.$ for each $\left.x \in e M e\right\}$. Then, by [4, Theorem 3.2.1] it suffices to show that $\{\mu, 1-\mu\} \subseteq S p\left(\left(\sigma^{\mu}\right)^{e}\right)$ for each $e$. However, if $f \in F_{\mu}$ then $\int f(t) \sigma_{t}^{\mu}\left(e S_{1} e\right) d t=0$ so $\int f(t) \mu^{-i t} d t=0$ and $\mu \in S p\left(\left(\sigma^{\mu}\right)^{e}\right)$; similarly, replacing $S_{1}$ by $S_{2}, 1-\mu \in S p\left(\left(\sigma^{\mu}\right)^{e}\right)$, as required.

Theorem 3.2. For each $0<\mu<\frac{1}{2}$ there exists a factor state $\psi_{\mu}$ of $0_{2}$ extending the trace on the Choi algebra. If there exist $0<\lambda<1$ and coprime natural numbers $n, m$ such that $\mu=\lambda^{n}$ and $1-\mu=\lambda^{m}$ then $\psi_{\mu}$ is of type III $_{\lambda}$; otherwise $\psi_{\mu}$ is of type $\mathrm{III}_{1}$.

Proof. Let $\psi_{\mu}$ be the factor state on $0_{2}$ defined above which, by Proposition 2.2 , extends the trace on the Choi algebra. Let $M=\pi_{\psi_{\mu}}\left(0_{2}\right)^{\prime \prime}$ and note that, by Lemma 3.1, $S(M) \neq\{0,1\}$ so, by [4, Theorem 3.4.1] $\psi_{\mu}$ will be of type III $_{1}$ if and only if $T(M)=\{0\}$ and will be of type III $_{\lambda}$ with $0<\lambda<1$ if and only if $T(M)=\{2 k \pi / \log (\lambda): k \in \mathbf{Z}\}$, where $T(M)=\left\{t \in \mathbf{R}: \sigma_{t}^{\mu}\right.$ is inner $\}$.

From [1, Theorem 4.3] $\sigma_{t}^{\mu}$ is inner if and only if $\mu^{-i t}=(1-\mu)^{-i t}=1$ i.e., if and only if $t=2 \pi k / \log (\mu)$ and $t=2 \pi k^{\prime} / \log (1-\mu)$ for some $k, k^{\prime} \in \mathbf{Z}$. Hence

$$
T(M)=\{2 \pi k / \log (\mu): k \in \mathbf{Z}\} \cap\left\{2 \pi k^{\prime} / \log (1-\mu): k^{\prime} \in \mathbf{Z}\right\} .
$$

If there exist $\lambda, n, m$ as in the statement of the theorem then, for each $t \in$ $T(M)$, there exist $k, k^{\prime} \in \mathbf{Z}$ with $t=2 k \pi / n \log (\lambda)=2 k^{\prime} \pi / m \log (\lambda)$. It follows that $k m=n k^{\prime}$ and, since $m, n$ are coprime, that $t \in\{2 k \pi / \log (\lambda): k \in \mathbf{Z}\}$. It is then easy to see that $T(M)=\{2 k \pi / \log (\lambda): k \in \mathbf{Z}\}$, so that $\psi_{\mu}$ is of type III $_{\lambda}$. To complete the proof, note that if $T(M) \neq 0$ then there exist nonzero $n, m \in \mathbf{Z}$ such that $2 n \pi \log (1-\mu)=2 m \pi \log (\mu)$; furthermore we can assume $n>0, m>0$ and that $n, m$ are coprime. Hence, letting $\lambda=\mu^{1 / n}$, there exists $0<\lambda<1$ and coprime natural numbers $n, m$ such that $\lambda^{n}=\mu$ and $\lambda^{m}=1-\mu$, as required.

Corollary. There exist infinitely many nonequivalent type III factor states of $\mathrm{O}_{2}$ extending the trace on the Choi algebra. 
Proof. For each $n>1$ the equation $\lambda+\lambda^{n}=1$ has a (unique) solution $\lambda_{n}$ in $\left(\frac{1}{2}, 1\right)$ : let $\mu(n)=1-\lambda_{n}$. Then $0<\mu(n)<\frac{1}{2}, \mu(n)=\lambda_{n}^{n}$ and $1-\mu(n)=\lambda_{n}$ so that $\psi_{\mu(n)}$ of type III $_{\lambda_{n}}$. Since $\lambda_{n} \neq \lambda_{m}$ whenever $n \neq m$ the states $\psi_{\mu(n)}$ for $n>1$ provide the required family.

The corollary has also been obtained in [9, Theorem 3.27], where a different but related construction produces extensions of type $\mathrm{III}_{\lambda}$ for each $0 \leq \lambda \leq 1$, and in [2, Theorem 3.2], where the states $\psi_{\mu}$ of the present paper are used. The result given in [2, Theorem 3.2] applies both to $\psi_{\mu}$ on $0_{n}$ and certain states on $K \otimes 0_{n}$; the part applying to $\psi_{\mu}$ is essentially equivalent to the case $m=n+1$ of Theorem 3.2 , so that the two results are very closely related. However the methods of proof are different: in [2] the type of $\pi_{\psi_{\mu}}\left(0_{n}\right)^{\prime \prime}$ is found by determining the asymptotic ratio set of a suitable crossed product (which is quite a lengthy calculation) whereas the present argument is a straightforward application, using results from [1,6], of the fundamental results of Connes on his invariants $S(M)$ and $T(M)$.

\section{ACKNOWLEDGMENT}

I would like to thank Dr. Rob Archbold for pointing out a minor error in a previous version of the paper and Professor Palle Jorgensen for his encouragement to publish this work.

\section{REFERENCES}

1. H. Araki, A. L. Carey, and D. E. Evans, On $0_{n+1}$, J. Operator Theory 12 (1984), 247-264.

2. R. J. Archbold, A. Lazar, S-K. Tsui, and S. Wright, Factor state extensions of type III, J. Funct. Anal. 95 (1991), 219-230.

3. M. D. Choi, A simple $C^{*}$-algebra generated by two finite order unitaries, Canad. J. Math. 31 (1979), 867-880.

4. A. Connes, Une classification des facteurs de type III, Ann. Sci. École Norm. Sup. (4) 6 (1973), 133-252.

5. J. Cuntz, Simple $C^{*}$-algebras generated by isometries, Comm. Math. Phys. 57 (1977), 173185.

6. D. E. Evans, On $0_{n}$, Publ. Res. Inst. Math. Sci. 16 (1980), 915-927.

7. A. Lazar, S.-K. Tsui, and S. Wright, Pure state extensions of the trace on the Choi algebra, Proc. Amer. Math. Soc. 102 (1988), 957-964.

8. G. K. Pedersen, $C^{*}$-algebras and their automorphism groups, Academic Press, New York, 1979.

9. J. Spielberg, Type III factor states on $\mathrm{O}_{2}$ which extend the trace on Choi's algebra, J. Operator Theory (to appear).

Department of Mathematics, La Trobe University, Bundoora 3083, Victoria, AusTRALIA 